\title{
Energy Spread Monitoring for the JLAB Experimental Program: Synchrotron Light Interferometers, Optical Transition Radiation Monitors and Wire Scanners ${ }^{1}$
}

\author{
Y.-C. Chao*, P. Chevtsov*, A. Day*, A.P. Freyberger*, R. Hicks*, M. Joyce* \\ and J.-C. Denard ${ }^{\dagger}$
}

* Jefferson Lab, 12000 Jefferson Avenue, Newport News, VA 23606

${ }^{\dagger}$ Synchrotron SOLEIL, 91192 CEDEX, France

\begin{abstract}
The hypernuclear physics program at JLAB requires an electron beam with small transverse size $(\sigma \sim 100 \mu m)$ and an upper limit on the RMS energy spread of $\frac{\delta E}{E}<3 \times 10^{-5}$. To measure and monitor these parameters, a beam size and energy spread measurement system has been created. The system consists of a set of wire scanners, Optical Transition Radiation (OTR) detectors, and Synchrotron Light Interferometers (SLI). The energy spread is measured via a set of wire scans performed at specific locations in the transport line, which is an invasive process. During physics operation the energy spread is monitored continuously with the OTR and/or the SLI. These devices are non-invasive [or nearly non-invasive in the case of OTR] and operate over a very wide range of beam energies $(1-6 \mathrm{GeV})$ and currents $(\sim 100 \mu \mathrm{A}$ down to few $\mu \mathrm{A})$. All components of this system are automated in an EPICS accelerator control environment. The paper presents our operational experience with the beam size and energy spread measurement system and its maintenance.
\end{abstract}

\section{INTRODUCTION}

The hypernuclear physics program at JLAB is attempting to measure very small mass splittings and widths of hypernuclear states. A $280 \mathrm{KeV}$ mass resolution is needed for the experimenters to observe the closely spaced mass states. The mass of the hypernuclear state is measured by the "missing mass" technique, where the four vectors of the incoming and scattered electron are measured and used to determine the mass of the hypernuclear state. The equation for the missing mass squared, $\left(M_{X}^{2}\right)$ is:

$$
M_{X}^{2}=m_{p}^{2}+2 m_{p}\left[E_{0}-E^{\prime}\right]+2 E_{0} E^{\prime} \cos (\theta)
$$

where $E_{0}$ is the energy of the beam, and $E^{\prime}$ is the energy of the $e^{-}$after scattering off the proton target, $m_{p}$. Energy spread of the incident electron beam will broaden/smear the $M_{X}^{2}$ distribution. Of the $280 \mathrm{KeV}$ resolution budget, $120 \mathrm{KeV}$ is allocated to energy

\footnotetext{
1 This work was supported by the U.S. Department of Energy under Contract No DE-AC05-84ER40150
} 
TABLE 1. Tabulation of the operating range and capabilities of the three devices used to measure the transverse beam width in the end station transport lines.

\begin{tabular}{cccc}
\hline device & current range & beam interaction & measures \\
\hline wire scanners/quad scan & $<20 \mu \mathrm{A}$ & invasive & $\sigma_{E} / E, \alpha, \beta, \gamma$ \\
\hline OTR & $8 \rightarrow 180 \mu \mathrm{A}$ & slightly invasive & $\sigma_{\text {beam }}$ \\
\hline SLI & $20 \rightarrow \infty \mu \mathrm{A}$ & non-invasive & $\sigma_{\text {beam }}$ \\
\hline
\end{tabular}

spread, which with $E_{0}=4 \mathrm{GeV}$ results in a requirement of:

$$
\frac{\sigma_{E}}{E_{0}}<3 \times 10^{-5}
$$

This paper describes the instrumentation that has been installed in the end station transport beam-lines to measure and monitor the energy spread during these experiments. The instrumentation includes wire scanners, optical transition radiation monitors and synchrotron light interferometers.

\section{MEASURING ENERGY SPREAD}

The transverse beam size, $\sigma_{\text {beam }}$, measured in a dispersive location has two sources:

$$
\sigma_{\text {beam }}=\sqrt{\sigma_{\beta}^{2}+\sigma_{\delta}^{2}}
$$

where $\sigma_{\beta}=\sqrt{\varepsilon \beta}$ is the betatron size and $\sigma_{\delta}$ is the size due to dispersion [1]. The energy spread is $\frac{\sigma_{E}}{E_{0}}=\frac{\sigma_{\delta}}{D}$, where $\mathrm{D}$ is the local dispersion. Ignoring the betatron contribution (which is safe to do when $\frac{\sigma_{\beta}}{\sigma_{\delta}}<<1$ ) the upper limit on the energy spread is:

$$
\frac{\sigma_{E}}{E_{0}}<\frac{\sigma_{\text {beam }}}{D} .
$$

When the beam has a small energy spread, as with the JLAB beam, $\sigma_{\beta}$ can be comparable to $\sigma_{\delta}$ and the betatron contribution to the transverse beam size must be taken into account to determine the central value of the energy spread.

In order to minimize the betatron contribution to the beam size, as well as the effect of incoming dispersion, a special optics was devised for the end station transport line to increase the dispersion from $4 \mathrm{~m}$ (nominal) to $8 \mathrm{~m}$ (high dispersion).

The transverse beam size is measured by wire scanners, optical transition radiation monitors and synchrotron light interferometers. The operating ranges and capabilities of the devices are listed in Table 1, and discussed in more detail in the following sections. 


\section{Wire Scanners}

Wire scanners, being the originally installed devices to measure transverse beam size, are numerous [there are 10 wire scanners in the end station $\mathrm{A}$ and $\mathrm{C}$ transport lines and five in the end station B transport line] and well understood devices [2]. The JLAB wire scanner design uses three $25 \mu \mathrm{m}$ W97Re 3 wires in vertical, horizontal and $45^{\circ}$ orientations. The wire position is correlated with the current induced on the wire from secondary emission or with the photomultiplier tube response due to the resulting shower from the electron scattering off the wire. Measuring the beam parameters with a wire scanner is limited to beam currents below $20 \mu \mathrm{A}$ and is invasive to the beam delivery.

The ten installed wire scanners ${ }^{2}$ allow the extraction of the beam Twiss parameters, $\alpha, \beta, \gamma, \varepsilon$, without having to adjust any quadrupole strengths [i.e. quad scan]. The software for obtaining and analyzing the wire scan data is broken into three steps:

1. Cycle individual wire scanners and fit the processed data to a Gaussian distribution with constant background. A 'best in show' wire scan is shown in Figure 1. All wire scan data are written to disk for further analysis and comparison with previous history.

2. Cycle all or a subset of wire scanners sequentially, verify the data integrity and summarize the results along with the quadrupole magnetic fields during the scans. A complete scan of all the wire scanners in the transport line takes about 30 minutes.

3. Take the accumulated wire scan data from the previous step and extract the Twiss parameters. Details of the Twiss parameter extraction can be found in Ref. [3].

\section{Optical Transition Radiation [OTR] Monitors}

Optical transition radiation[4] monitors have been installed at selective locations in the transport line to complement the wire scanners. The OTR monitors measure the transverse beam size by imaging the OTR on CID camera and analyzing the horizontal and vertical projections. A carbon foil of $1 \mathrm{~cm} \times 1 \mathrm{~cm}$ area and $0.2 \mu \mathrm{m}$ thickness ${ }^{3}$ presents such a negligible material to the beam that beam can be delivered to the end station while the OTR foil is inserted in the beam line. Neutral density filters are used to adjust the light intensity within the optimum range for the camera. The OTR operates with CW beam with current from $10 \mu \mathrm{A}$ up to $180 \mu \mathrm{A}$. See Ref. [5] for details.

The very thin carbon foils are mounted on a three sided frame. OTR emitted in the forward direction is used to form the beam image. Forward OTR distribution is not affected by local shape of the foil as in the case of backward OTR. Figure 2 shows the mechanical layout of the OTR.

${ }^{2}$ Only four non-degenerate measurements of the transverse beam size are needed to extract the Twiss parameters.

${ }^{3}$ Foils are obtained from Goodfellow Corp., www.goodfellow.com 


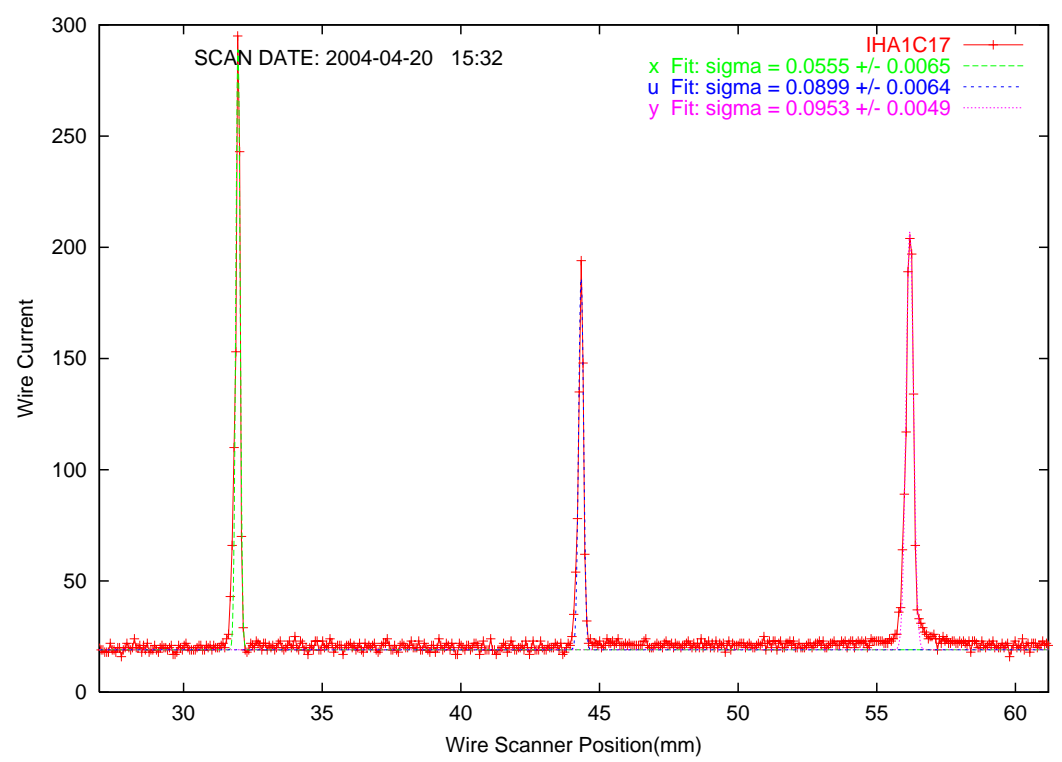

FIGURE 1. Wire Scan data and fit results. The three peaks correspond to the three wires, horizontal, $45^{\circ}$, and vertical orientations. Each peak is fit to a Gaussian distribution with a constant background.



FIGURE 2. Mechanical schematic of the forward optical transition radiation monitor. The $0.2 \mu \mathrm{m}$ carbon foil and mirror are mechanically coupled.

The beam size as measured by the OTR is calibrated by comparison with the beam size as measured by the wire scanners. This calibration is done at $8 \mu \mathrm{A}$ of beam current 


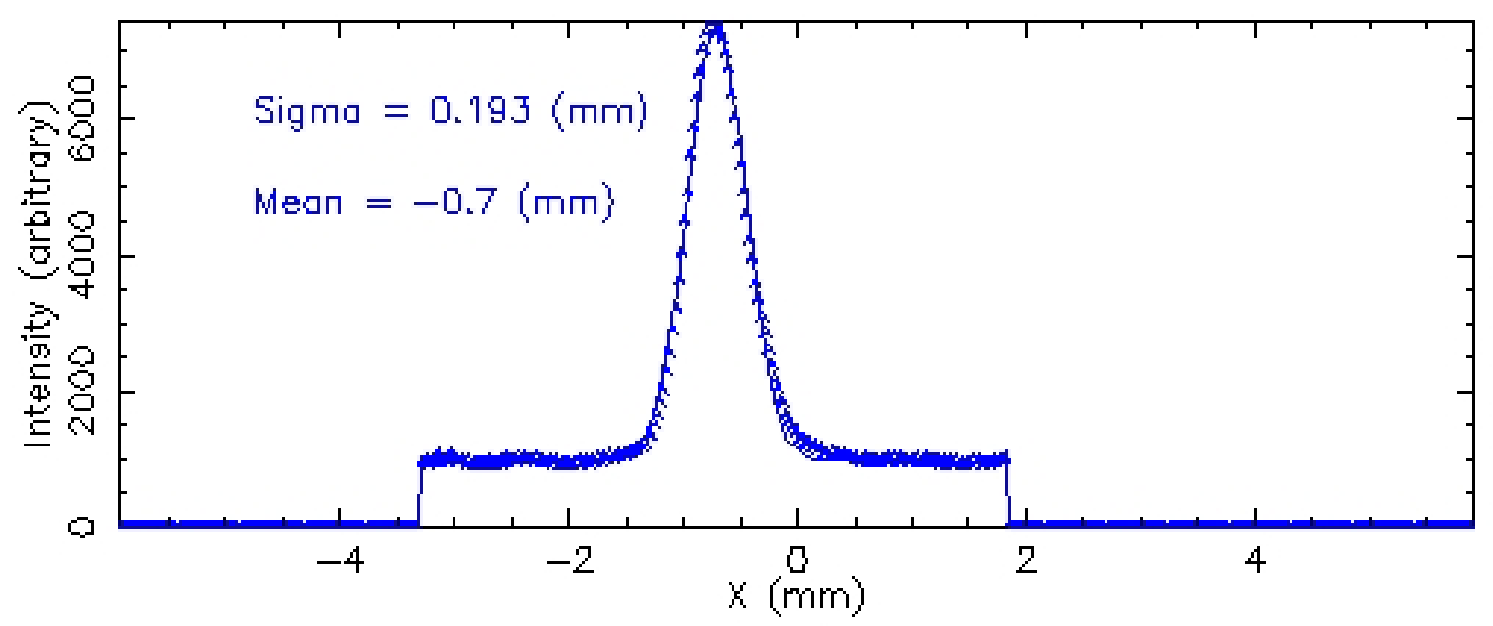

FIGURE 3. Typical OTR horizontal projection. The points are the data from the CID camera, the blue line and values for $\sigma$ and position are the result of a fit to the data.

and different beam sizes are obtained by varying quadrupole magnet field strengths ${ }^{4}$.

Figure 3 shows the horizontal beam profile as measured by the OTR. The OTR images are analyzed in real time. Horizontal and vertical projections are fit to a Gaussian distribution with a constant background.

\section{Synchrotron Light Interferometer [SLI]}

The use of synchrotron light interferometry makes it possible to measure the very small transverse beam size in a completely non-invasive manner. SLI installations at storage rings have an abundant amount of synchrotron light due to the large stored beam current. The lower JLAB beam current is advantageous in that beam heating of the SLI optical components is no longer an issue as it is at storage rings. However, at these lower beam currents the intensity of the light is so diminished that a cooled CCD camera ${ }^{5}$ is necessary to image the interferogram.

The difference in light intensity and the choice of CCD camera is the major difference between the JLAB SLI and that of T. Mitsuhashi at KEK [6]. A sketch of the components used to make a SLI are shown in Figure 4 and details of the design are found in Ref. [7].

\footnotetext{
${ }^{4}$ We have found that the optical components get dusty which introduces a changing point source term [offset]. This requires an OTR calibration and/or cleaning to be performed on a periodic basis. The source of the dust is a mystery since the optical components are in a closed system.

5 Santa Barbara Instrument Group, Astronomical CCD cameras, www . sbig • com.
} 


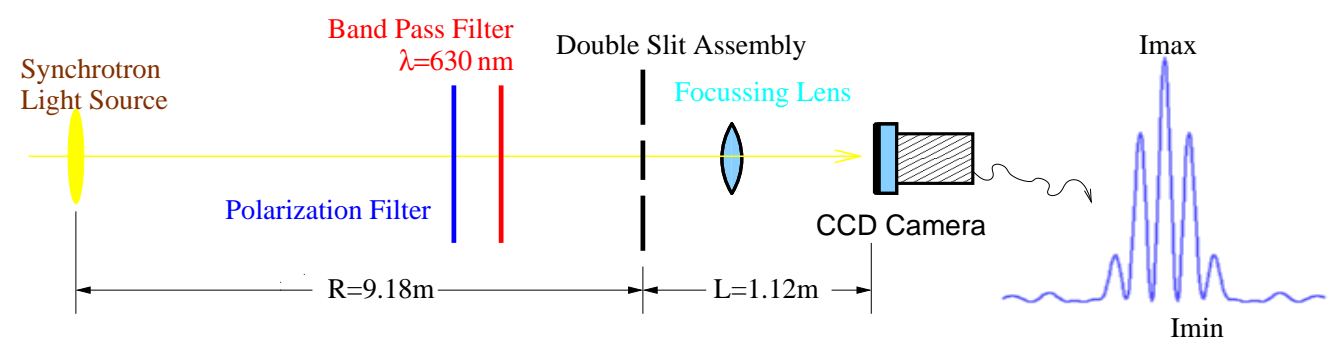

FIGURE 4. Cartoon schematic of the synchrotron light interferometer.

The beam size is a function of the visibility, $\bigvee$, of the interference pattern:

$$
\mathrm{V}=\frac{I_{\max }-I_{\min }}{I_{\max }+I_{\min }},
$$

where $I_{\max }\left(I_{\min }\right)$ is the maximum (minimum) of the interference pattern. Since $\bigvee$ is a ratio, most systematics involved in digitization cancel. However, noise terms contribute to the sum [denominator] leading to a reduced $\bigvee$, or larger calculated beam size and therefore must be kept small.

For a Gaussian beam distribution the beam size is found to be:

$$
\sigma_{\text {beam }}=\frac{\lambda_{0} R}{\pi d} \sqrt{0.5 \ln (1 / \bigvee)},
$$

where $\lambda_{0}$ is the wavelength of the bandpass filter, $R$ is the distance from the light source to the double slit assembly, $d$ is the slit separation and $\bigvee$ is the measured visibility.

The JLAB SLI uses a grid pattern for the double slit assembly. This allows the simultaneous measurement of horizontal and vertical beam sizes. Figure 5 shows the real-time display of the interferogram data and fits to the data. The data update rate is a function of the beam current; as fast as 0.5 seconds for $120 \mu \mathrm{A}$ and tens of seconds for beam currents less than $20 \mu \mathrm{A}$. Figure 6 is a plot of the beam sizes as measured by the OTR and SLI. There is an discrepancy with the larger beam sizes that is presently being investigated.

\section{CONCLUSIONS}

A suite of instrumentation to measure and continuously monitor the energy spread at the end station transport lines has been presented. Two transport lines have been equipped with the full compliment of instrumentation. Figure 7 shows the stability of the energy for several days of operation. Initially the energy spread was near the specification of $\frac{\sigma_{E}}{E}<3 \times 10^{-5}$. Improving the operation of the RF cavities and general machine setup resulted in a dramatic improvement of energy spread, seen on the plot as the drop in energy spread mid-day on April 23. The continuous operation of the SLI and OTR have proved to be useful in identifying components contributing to the measured energy spread. 


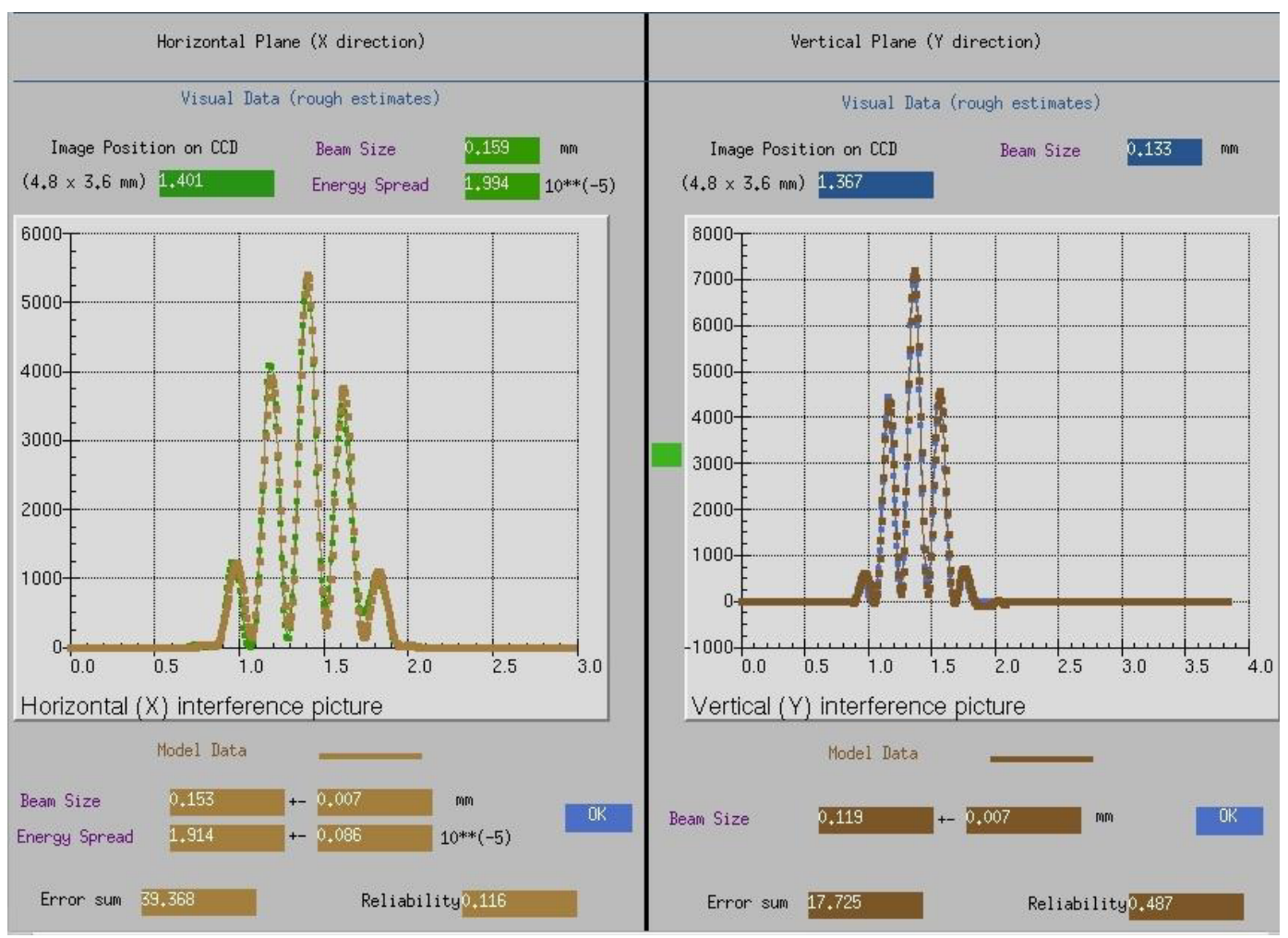

FIGURE 5. Control screen for the SLI showing the horizontal and vertical interferograms. The green (blue) points are the horizontal (vertical) data and the brown points are the result of a fit to the data. The results for the beam size above the interferogram are the result of a simple search algorithm to determine $I_{\max }$ and $I_{\min }$. The values below the interferogram result from a fit to the expected functional form.

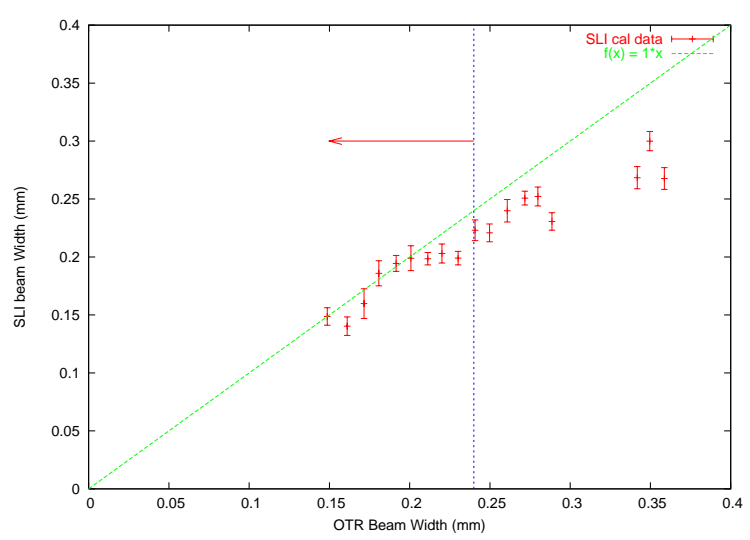

FIGURE 6. Plot of the beam width as measured by the SLI vs OTR. The green dashed curve is a line with slope of one and intercept of zero.

\section{ACKNOWLEDGMENTS}

The authors thank the JLAB operations staff for support during commissioning the devices. 


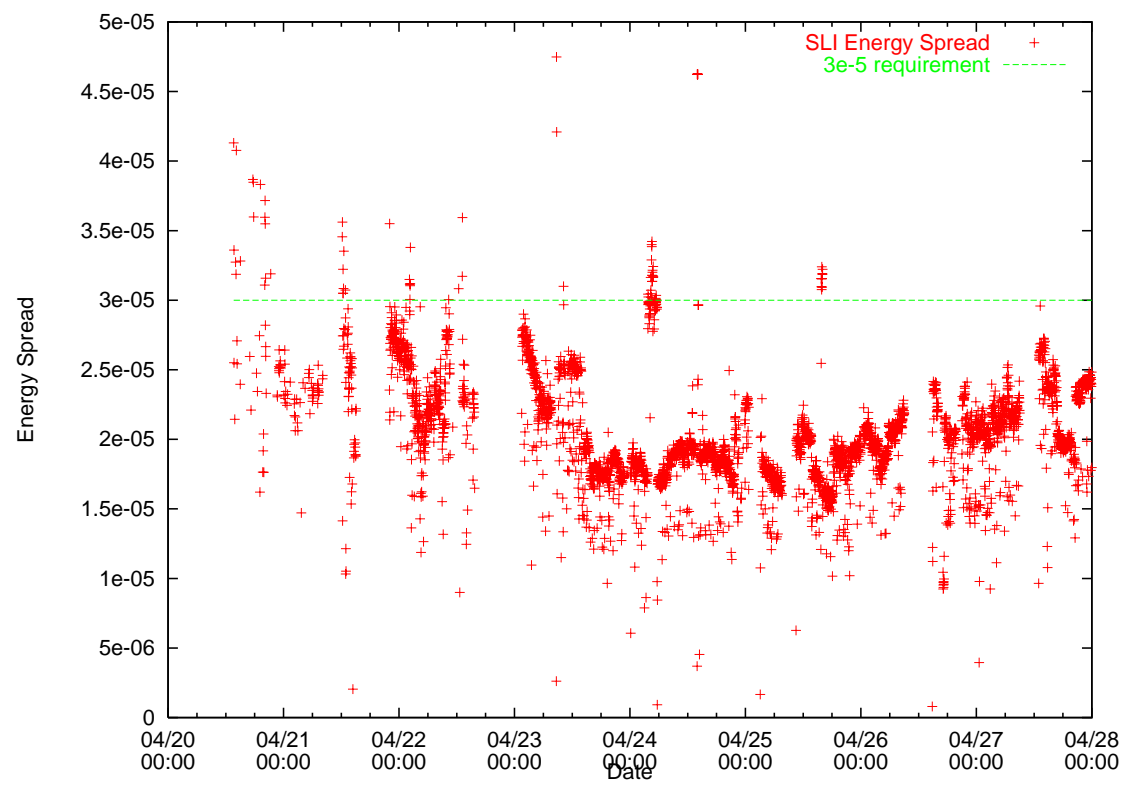

FIGURE 7. Plot of the SLI measurement of energy spread as a function of time.

\section{REFERENCES}

1. M.G. Minty and F. Zimmerman, Measurement and Control of Charged Particle Beams, SpringerVerlag, Berlin, 2003, pp. 163.

2. M. Plum, "Interceptive Beam Diagnostics - Signal Creation and Materials Interactions", in these proceedings.

3. Y-C. Chao, "Measuring and Matching Transport Optics at Jefferson Lab", in Proceedings of 2003 PAC, http: //accelconf.web.cern.ch/accelconf/p03/PAPERS/TOPB011.PDF.

4. V.L. Ginzberg and V.N. Tsytovich, Transition Radiation and Transition Scattering, Adam Hilger, Bristol, 1990, 448 p.

5. J-C. Denard et al., "High Power Beam Profile Monitor with Optical Transition Radiation", in Proceedings of the 1997 PAC, http://accelconf.web.cern.ch/accelconf/pac97/ papers/pdf/5P062.PDF.

6. T. Mitsuhashi, "Faraday Cup Award Talk: 12 Years of SR Monitor Development at KEK", in these proceedings.

7. P. Chevtsov et al., "Synchrotron Light Interferometer at Jefferson Lab", in Proceedings of the 2003 PAC, http://accelconf.web.cern.ch/accelconf/p03/PAPERS/WPPB068.PDF. 